- Foreign bodies often induce reparative granuloma formation or suppuration but can eventually have a different course, making diagnosis difficult.

- Although rare, maxillofacial foreign bodies can be misdiagnosed as odontogenic lesions, so correct diagnosis is essential to performing the appropriate treatment.

- Scanning electronic microscopy can be a useful method in identifying the origin of foreign bodies.

\title{
Unusual wooden foreign body in the palate
}

\author{
A. M. P. Soubhia, ${ }^{1}$ A. C. P. Ribeiro, ${ }^{2}$ L. D. Martins, ${ }^{3}$ A. R. S. Silva ${ }^{4}$ and M. A. Lopes ${ }^{5}$
}

We report a case of a palatal calcified foreign body simulating an odontogenic lesion. Surgical exploration revealed a calcified mass that was analysed under light microscopy and identified as a vegetal foreign body. Further scanning electron microscopy analysis revealed that the foreign body was a piece of wood. Hard palate foreign bodies have been reported previously, however, it seems that this is the first case of its kind.

\section{INTRODUCTION}

Foreign bodies of the hard palate are considered rare and may be present in a variety of ways. Nut shells, clothing buttons, plastic emblems, screw covers, haemostatic pack, resin tube and a gutta percha have already been described as palatal foreign bodies and the majority of these cases involve infants. ${ }^{1,2}$ Often the resulting lesions can lead to a misdiagnosis of odontogenic cysts or tumours. ${ }^{1,2}$

Wooden foreign bodies have been previously reported in soft tissue. ${ }^{3-}$

${ }^{5}$ However, to the best of the authors' knowledge, there is no paper describing an intraosseous foreign body associated with the toothpick.

\section{CASE REPORT}

A 53-year-old man attended a private dentistry clinic complaining of a

\footnotetext{
1.2Department of Pathology and Clinical Propedeutic (Oral Pathology), São Paulo State University (UNESP), Araçatuba, São Paulo, Brazil; ${ }^{3}$ Private Practice, Ponta Grossa, Paraná, Brazil: $4,5^{*}$ Department of Oral Diagnosis (Semiology), Piracicaba Dental School, State University of Campinas (UNICAMP), Piracicaba, São Paulo, Brazil *Correspondence to: Dr Marcio Ajudarte Lopes Email:malopes@fop.unicamp.br
}

swelling in the incisive papilla. His history included an episode of traumatic laceration of the incisive papilla with a toothpick 15 years previously, which healed on its own. The patient continued with his habit of using a toothpick at least three times a day.

Intraoral examination revealed only an asymptomatic swelling of the incisive papilla, with a diameter of $1 \mathrm{~cm}$. A standard maxillary occlusal radiograph revealed the presence of a radiopaque structure circumscribed by a radiolucent area and located between the roots of the upper central incisors (Fig. 1). With these clinical and radiographic features, the main provisional diagnosis was supernumerary teeth and an odontogenic tumour, particularly an odontoma.

Surgical exploration under local anaesthesia was performed and a hard material circumscribed by fibrous tissue was obtained. Haematoxylin-eosin staining assessed by light microscopy showed an amorphous material involving a foreign body composed of fibres probably of vegetal origin (Fig. 2). Adjacent soft fibrous tissue had few chronic inflammatory cells. In order to confirm the origin of the foreign body a SEM examination (JEOL, JSM-5600LV, Scanning Electron Microscope, Japan) using a $5 \mu \mathrm{m}$ par- affin section sputtered with gold was performed. The specimens were mounted on aluminium stubs and observed at low vacuum with back-scattered electron images, revealing a vegetal arrangement structure consistent with longitudinal fibres of wood (Fig. 3). The patient is in follow-up for about eight months with no further problems.

\section{DISCUSSION}

Intraosseous hard palate foreign bodies are an uncommon condition and only a small number of cases have being reported; almost all of them in paediatric patients. These foreign bodies are usually

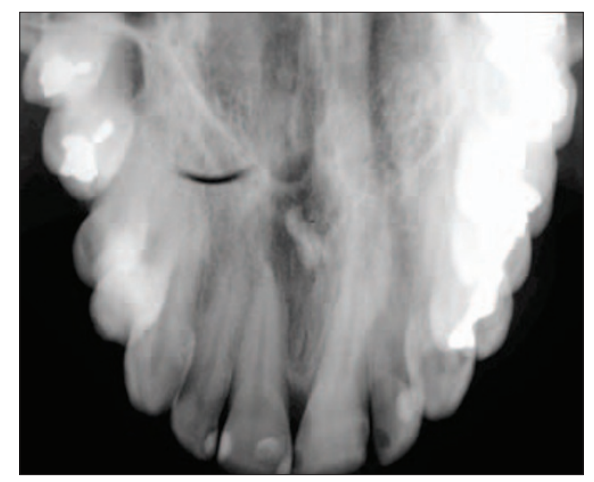

Fig. 1 Standard maxillary occlusal radiograph demonstrating a radiopaque structure circumscribed by a radiolucent area between the roots of the upper central incisors (a semicircular radiolucent artifact caused by finger nail can be seen on the left) 


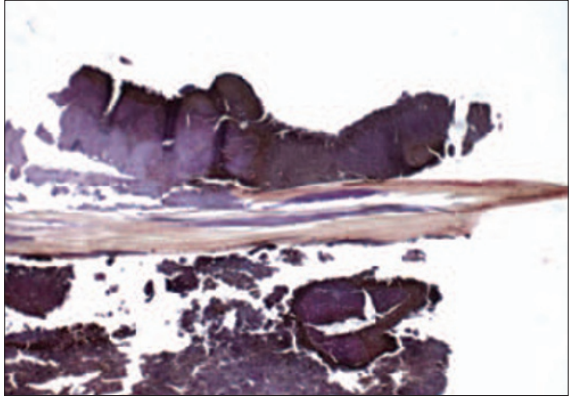

Fig. 2 Amorphous material involving a foreign body composed of lengthen and thin fibers (Haematoxylin and eosin, original magnification, $x$ 5)

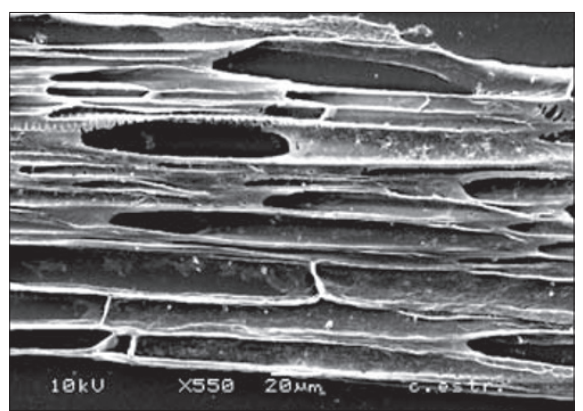

Fig. 3 SEM examination revealing a vegetal arrangement structure consistent with longitudinal fibres of wood

due to accidental implantation or to iatrogenic causes; the most common referring diagnosis in the literature for these cases is a suspected odontogenic tumour. ${ }^{1,2}$

Although it is extremely rare, wood can be considered an ideal medium for microbial agents because of its organic nature and porosity, and it is capable of inducing a reparative granuloma formation, suppuration or chronic inflammation. Infection is more frequent, however, and sometimes wood may also lead to an aseptic foreign body reaction. ${ }^{4,5}$ Both types of inflammation demand complete surgical removal of the retained foreign body in order to cure the symptoms. ${ }^{5}$

Foreign bodies often incite a chronic inflammatory reaction with the deposition of mineral salts, similar to other types of calculi formation such as sialolithiasis, rhinolith and tonsillolith. ${ }^{6}$

Since wood is an organic material with low density, plain radiographs are almost unable to detect their presence in soft tissues and an ultrasound is the best imaging modality for detection of suspected wooden foreign bodies in soft tissues. ${ }^{4,5,7,8}$ However, when wood induces calcification, it can be detected by conventional radiographic exams as in the reported case. On the other hand, the toothpick fragment could only be observed by macroscopic or microscopical analyses.

A clinicopathological correlation, especially by using the SEM analysis, provided enough information in this case to support the fact that the intraosseous foreign body was due to the patient's habit of using a toothpick and the accidental implantation of a toothpick fragment 15 years previously.

Considering the clinical features, particularly the location and the absence of complaint, and the radiographic aspects, many other lesions could be considered in the differential diagnosis such as a supernumerary tooth, rhinolith or odontogenic tumours. In the last group odontoma, calcifying cystic odontogenic tumour, adenomatoid odontogenic tumour and calcifying epithelial odontogenic tumour are the most probable. ${ }^{9}$

Odontogenic tumour, like foreign body of the hard palate, is an uncommon diagnosis. Dental practitioners should keep both hypotheses on a differential diagnosis because they are often misdiagnosed and have totally separate treatments and prognoses.

The authors gratefully acknowledge Adriano Luis Martins from the Department of Oral Diagnosis, Piracicaba Dental School - UNICAMP, for his SEM examination.

1. Tseng E, Woolley A L. Foreign body simulating a hard palate lesion in a child. Int J Pediatr Otorhinolaryngol 1996; 38: 169-174.

2. Jong A L, Moola F, Kramer D, Forte V. Foreign bodies of the hard palate. Int J Pediatr Otorhinolaryngol 1998; 43: 27-31.

3. Gallagher C, Sleeman D. Interesting case: when you can't see the wood: a foreign body pitfall. Br J Oral Maxillofac Surg 2006; 44: 93.

4. Auluck A, Behanan A G, Pai K M, Shetty C. Recurrent sinus of the cheek due to a retained foreign body: report of an unusual case. Br Dent J 2005; 198: 337-339.

5. Krimmel M, Cornelius C P, Stojadinovic S, Hoffmann J, Reinert $\mathrm{S}$. Wooden foreign bodies in facial injury: a radiological pitfall. Int J Oral Maxillofac Surg 2001: 30: 445-447.

6. Barros C A, Martins R R, Silva J B et al. Rhinolith: A radiographic finding in a dental clinic. Oral Surg Oral Med Oral Pathol Oral Radiol Endod 2005; 100: 486-490.

7. Peterson J J, Bancroft L W, Kransdorf M J. Wooden foreign bodies: imaging appearance. AJR Am J Roentgenol 2002; 178: 557-562.

8. Oikarinen K S, Nieminen T M, Makarainen $H_{\text {, }}$ Pyhtinen J. Visibility of foreign bodies in soft tissue in plain radiographs, computed tomography, magnetic resonance imaging, and ultrasound. An in vitro study. Int J Oral Maxillofac Surg 1993; 22: 119-124.

9. Barnes L, Eveson J W, Reichart P, Sidransky D. World Health Organization classification of tumours. Pathology and genetics of head and neck tumours. Lyon: IARC Press, 2005. 